\title{
Gestión del Conocimiento desde una Mirada Compleja y Transdisciplinar en la Universidad Privada San Francisco de Asís - El Alto
}

\author{
Knowledge Management from a Complex and Transdisciplinary perspective at the San \\ Francisco de Asís Private University - El Alto
}

Carlos Jorge Landaeta-Mendoza ${ }^{1}$

\section{ఠ} EDICIÓN: ®E-CIVTAC

Recibido: $8 /$ julio/2020

Aceptado: 28/julio/2020

Publicado: 25/septiembre/2020

${ }^{1}$ Bolivia

\section{IIIIInstitución \\ IUniversidad Privada San Francisco de Asís}

\section{Correo Eletrónico}

1clandaeta@usfa.edu.bo

\section{ORCID}

1https://orcid.org/0000-0003-0297-7029

\section{Citar así: GCAPA / IEEE}

Landaeta-Mendoza, C. (2020). Gestión del Conocimiento desde una Mirada Compleja y Transdisciplinar en la Universidad Privada San Francisco de Asís - El Alto. Revista Tecnológica-Educativa Docentes 2.0, 9(2), 79-87. https://doi.org/10.37843/rted.v9i2.148

C. Landaeta-Mendoza, "Gestión del Conocimiento desde una Mirada Compleja y Transdisciplinar en la Universidad Privada San Francisco de Asís - El Alto", RTED, vol. 9, n. ${ }^{\circ}$, pp. 79-87, sep. 2020.

\section{Resumen}

La dinamización del contexto universitario a partir del proceso de Gestión del conocimiento en la relación contexto/realidad académica con el contexto/realidad sociocultural, conlleva a que los subsistemas internos que conforman el entorno universitario puedan promover procesos innovadores que faciliten la gestión de recursos y estrategias en pro de la gestión del conocimiento. La investigación revaloriza el conocimiento académico, inmerso en un conjunto de factores que activan el desarrollo de la sociedad, donde se dinamizan procesos de transformación de la sociedad en pro de prevenir riesgos psicosociales con acciones prácticas. implicando a toda una comunidad universitaria. La investigación tomó en cuenta el paradigma del pensamiento complejo, basado en el estudio de caso como método de investigación, utilizando la posición estratégica del investigador. La implicación de toda una comunidad universitaria, contribuye a la gestión del conocimiento que a la vez es un eje articulador que permite una amplitud en la toma de decisiones a nivel interno y posterior vinculación continua con la sociedad, convirtiendo a la universidad como espacio de referencia, gracias al vínculo contexto/realidad académica y contexto/realidad sociocultural mediados por el proceso de gestión del conocimiento basada en innovación, creatividad y promoción de procesos participativos. La gestión del conocimiento debe ser promovido de forma continua por las autoridades, acciones que facilita una gestión continua, participativa y activa; logrando implicar a todos los subsistemas que conforma la universidad, teniendo como resultado el mejoramiento de los vínculos y tipos de comunicación entre los implicados.

Palabras clave: Proceso de gestión del conocimiento, contexto/realidad sociocultural, contexto/realidad académica, innovación, dinamización.

\section{Abstract}

The dynamization of the university context from the knowledge management process in the relationship context / academic reality with the context / sociocultural reality, leads to the internal subsystems that make up the university environment can promote innovative processes that facilitate the management of resources and strategies in favor of knowledge management. The research revalues ??academic knowledge, immersed in a set of factors that activate the development of society, where processes of transformation of society are stimulated to prevent psychosocial risks with practical actions. involving an entire university community. The research considered the paradigm of complex thinking, based on the case study as a research method, using the strategic position of the researcher. The involvement of an entire university community contributes to the management of knowledge that is also an articulating axis that allows a breadth of internal decision-making and subsequent continuous linkage with society, making the university a reference space Thanks to the link between the academic context / reality and the sociocultural context / reality mediated by the knowledge management process based on innovation, creativity and the promotion of participatory processes. Knowledge management must be promoted continuously by the authorities, actions that facilitate continuous, participatory and active management; managing to involve all the subsystems that make up the university, resulting in the improvement of the links and types of communication between those involved.

Keywords: Technology, education, TAC, teaching, technoemotional. 


\section{Introducción}

La formación actual de los docentes universitarios, en muchos casos se centra en procesos formativos basados en lo teórico, en generación de esquemas cognitivos provenientes de textos, libros, cursos, entre otros. Donde a pesar de estar en pleno siglo XXI, algunos continúan siendo meros transmisores de la información, sin dar pie al prenderanalizar-debatir-reflexionar, que le permita desarrollar en los estudiantes habilidades, destrezas profesionales, basadas en la secuencia: obtencióndesestructurar-generación-aplicación-apropiación, del conocimiento facilitando.

El reto de la formación de profesionales debería inclinarse en que sus actores, en especial los docentes, aprendan a gestionar el conocimiento, pasando de la mera transmisión de información a religar, integrar, complementar, contextualizar, distinguir, ubicar, entre otros. Esto puede constituirse, en una estrategia importante e interesante, basada en los planteamientos por Morin (2005) los cuales podrán vincular y afrontar la incertidumbre, comprender los acontecimientos que se suscitan en los subsistemas externos e internos de la realidad en la que se encuentran. Contemplando al docente como un edificador de formas aptas para el desarrollo de la sociedad en general (Panza, 2020) cuya labor debe estar focalizada metafóricamente desde las acciones como alfarero, donde sus manos van delineando rasgos definitorios del ser como profesional el futuro.

Existe la necesidad y pertinencia de fomentar nuevas formas de conocimiento, con énfasis no solo en acumulación de conocimientos, sino en contextualizar y analizar el conocimiento, una prospectiva donde el docente reinterprete y reintegre los saberes dispersos en distintas disciplinas, relacionando el contexto académico con el contexto sociocultural.

Este planteamiento relacional entre el contexto/realidad académica y el contexto/realidad sociocultural, basados en procesos de gestión del conocimiento, se contemplan acciones que permitirán dinamizar el desarrollo del aprendizaje, generando diversos patrones de interacción social de tipo indexical con la realidad (Sotolongo, 2013) erigido sobre la base de: el involucramiento del futuro profesional con otras personas concretas y específicas, plasmación de sus conocimientos a través de prácticas de un determinado contenido.

Morin (2003) en su libro, el Método V "La humanidad de la humanidad" define al ser humano como un todo, no fragmentado, lo presenta como un ser conceptualizado, compactado en cuatro grandes componentes: en sus componentes biológicos, culturales, sociales e individuales como un ser único, cuyo escenario es la totalidad del universo y su gran laboratorio de observación es el cosmos en general. Es decir, lo presente a través de sus diferentes procesos: sus dos infinitos: cosmologías microfísicas; sus diferentes procesos biológicos, prehistóricos, ecológicos, de su destino sociales e históricos y el de la expresión del alma: el arte, la poesía, la pintura.

Desde un enfoque integrador partiendo de la Psicología, estos elementos componen la misma esencia del ser humano. Los procesos psicológicos superiores psicobiológicos abarcan la inteligencia, el aprendizaje - memora, la motivación - emoción, el pensamiento - lenguaje, la sensación - percepción, la atención - concentración. Se trata de procesos interconectados que proporcional a la persona de un funcionamiento cognitivo, a través del cual puede adaptarse al mundo externo.

Los procesos psicológicos, en el desarrollo de la persona, a lo largo de su vida, abarcan respuestas ante la angustia, instauran los componentes del temperamento, el carácter y la personalidad. Se trata, también, de procesos interconectados que dotan al individuo de un funcionamiento psicosocial a través del cual puede interactuar con los demás seres de su entorno. Los aportes que Morín (2003), basados en su texto Método V, expresan la interconectividad del pensamiento con la ciencia e identidad humana, cuya visión de eco educación coincide con la visión planetaria de Morín, alcanzable a través de la renovación del pensamiento actual, trocándolo por la mejor respuesta: el pensamiento complejo.

En relación con el proceso de gestión de recursos y estrategias que se dan en el contexto/realidad académica con el contexto/realidad sociocultural, desde los subsistemas relacionados con la realidad universitaria, se encuentran problemas entre los que se destacan los organizacionales y los metodológicos, los cuales tienen que ver directamente con los inconvenientes y retos que pasan los nuevos profesionales al incursionarse a la vida laboral, donde tienen dificultad en trabajar en equipo, sus conocimientos se centran en lo teórico, no pudieron 
relacionar, religar, aplicar, entre otros. La realidad en que se encuentran o se encontraran interactuando, donde hay un quiebre en el modelo "aula mente social" (Gonzales, 2013), generando en el nuevo profesional, dificultad para que se interrelacione con cada uno de los subsistemas.

A todas estas problemáticas, se suman la disminución del valor que se da a la adquisición de nuevos conocimientos, disminución que no solamente está presente en los estudiantes, sino también en los docentes y en todas las personas que están implicadas en los subsistemas educativos universitarios, existiendo un quiebre entre los fines en que debería perseguir la universidad, con lo que es la gestión del conocimiento.

La gestión del conocimiento precisa de un conjunto de acciones organizadas (López, 2006) y de un trabajo del intelecto creativo. Para su aplicabilidad es necesario la planeación, dirección, ejecución, seguimiento y evaluación de cada acción estratégica. También es necesario considerar la oportunidad del momento, los recursos y el espacio de implantación. Muchas veces el proceso de gestión del conocimiento se lo aborda desde la simplicidad, desde enfoques tradicionales, reduccionistas, ya que existen tendencias de que las personas que estén implicadas en los subsistemas educativos, en vez de generar acciones en pro de la gestión del conocimiento, se dedican a gestionar de información, lo que lleva a que se lo aborde al conocimiento, como una simple información.

La existencia de poca vinculación entre el docente, con el entorno administrativo universitario, que podría coadyuvar a facilitar procesos de gestión en pro de promover procesos prácticos, acompañados con investigación, dificulta la concreción de acuerdos, convenios para la apertura de espacios, en donde se puede desarrollar procesos prácticos e investigación. Ante esta realidad, muchos docentes no contemplan procesos de vinculación práctica de su materia con la realidad sociocultural.

Por lo que es menester, desde las relaciones contexto/realidad académica - contexto/realidad sociocultural, revalorizar el conocimiento académico, inmerso en el conjunto de factores que activan del desarrollo de la sociedad, donde los mismos implicados en los procesos (docentes - estudiantes, comunidad universitaria), van dinamizando procesos de transformación de nuestra sociedad en pro de prevenir algunos riesgos psicosociales.
No obstante, estos cambios algunas veces se caracterizan por su velocidad, generando dificultades a la hora de enfrentarlos, donde inclusive los mismos promotores, organizadores (los docentes) no pueden dar respuesta a estos por falta de experticia, práctica, experiencias (ya que ellos solamente se dedican a los procesos de enseñanza, basada en la transmisión de informaciones, dejando de lado la transmisión de conocimientos, frutos de su propia experiencia).

\section{Metodología}

La presente investigación, toma en cuenta el paradigma del pensamiento complejo, en donde la complejidad se encuentra presente, desde la comprensión de lo que es el proceso de gestión del conocimiento, hasta los componentes del objeto de estudio: Universidad Privada San Francisco de Asís, contemplando todos sus elementos, como éstos se relacionan, religan e influyen entre sí.

La presente investigación se desarrolló basada en el estudio de caso como método de investigación (Rodríguez, 1999), porque permite examinar de manera detallada, comprehensiva y sistemática los diferentes aspectos relacionados con omnijetividad, en éste caso los procesos de gestión del conocimiento entre el contexto/realidad académica con el contexto/realidad sociocultural, utilizando para ello la posición estratégica del Investigador, como Director Académico de la Universidad Privada San Francisco de Asís

\section{Resultados}

Para el presente estudio se consideró a la Universidad Privada San Francisco de Asís, en su Subsede El Alto, las acciones desarrolladas no llega a desplazar los procesos que se desarrollaron con anterioridad dentro de la universidad, más al contrario, se instalan junto a ellos en una coexistencia que dio lugar a nuevas formas de administración educativa. Propuesta que se caracteriza por una transformación total en la Gestión administrativa y académica, basada en tres áreas de intervención, ver figura 1 . 


\section{Figura 1}

Áreas de intervención transfactorial

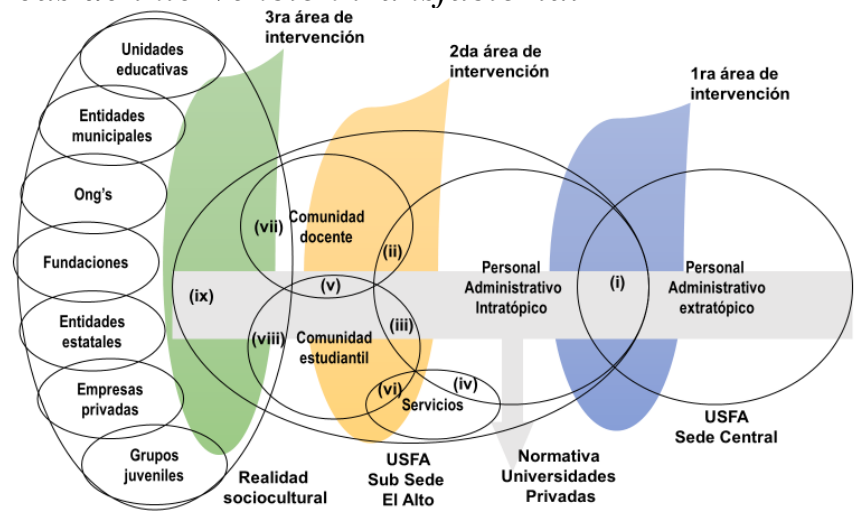

Nota. Gestión administrativa y académica, basada en tres áreas de intervención. Elaboración propia (2018).

La primera está centrara para todas aquellas personas administrativas que están a cargo tanto en gestión académica como administrativa, contemplando una relación continua entre el personal administrativo intratópico como extratópico, basadas en la dinamización entre todas las personas, generando acuerdos y herramientas que facilitan la gestión. Como segunda área de intervención se contempla el vínculo continúo entre el personal administrativo intratópicos con el personal de servicios, comunidad estudiantil y en especial con la comunidad docente, a partir del cual se van cambiando nuevos roles, promoviendo procesos investigativos continuos, el involucramiento a la realidad social, capacitaciones internas como acciones relacionadas con el impulso de capacitaciones externas, apoyando la participación y la responsabilidad social empresarial, un aspecto muy importante fue la integración familiar.

Se tiene como tercera área de intervención la vinculación entre comunidad docente/ estudiantil con la sociedad. Se fueron creando diversas reuniones de coordinación con fundaciones ONG, alcaldías con el fin de promover acuerdos y actividades conjuntas, se entablaron nuevas relaciones con empresas privadas, a partir del cual se generaron carreras y capacitaciones tanto a su personal, como también capacitaciones a los estudiantes, en función a la especificidad institucional. Se fomentaron reuniones con sociedad civil en general, en las que se promocionaron actividades culturales, artísticas, así también se participaron en ferias y otro tipo de eventos, en donde toda la comunidad universitaria estaba implicada, finalmente, a partir de ésta 3era área se promovió la participación en redes interinstitucionales.

Todas las acciones vinculadas con el proceso de intervención basada en la vinculación entre el contexto/realidad sociocultural con el contexto/realidad académica por medio de la gestión del conocimiento, permitió reestructurar todo el proceso administrativo y académico. El proceso de intervención práctica, que se realizó en la presente investigación, contempló nueve procesos transdiciplinarios, caracterizados por su dinamismo y promoción de la participación de todas las personas implicadas en el proceso, con cada uno de ellas se realizaron acciones en pro de facilitar la gestión del conocimiento, dejando de lado lo simplista: que mencionaría que solamente el docente es el responsable, dando pie a lo transdiciplinario, a lo holístico e integral, donde todos los implicados, aportan a una gestión del conocimiento que permita el desaprender-reaprender y aprender en los estudiantes como en ellos mismos.

Estos nueve procesos desarrollados fueron:

1. Reuniones con gestión de procesos entre el personal administrativo intratópico, de la misma Universidad Usfa El Alto, así como también reuniones y gestión con el personal administrativo de la Sede Central de la Universidad, representado por las Autoridades Nacionales: Rectorado y Vicerrectorado, vinculando acciones con los respectivos directores de carrera. Reuniones que buscaban, en primera instancia conocer el tipo de gestión de conocimiento que desarrollaban, así como también conocer algunos reglamentos, normativas que permitan justificar algunas acciones que se tenía en mente, en pro de dinamizar los procesos de aprendizaje-enseñanza dentro de la universidad.

Es en estas reuniones donde se identificaron que no se contaban con políticas específicas que permitan incentivar la autoformación docente ni motivación por un mejor servicio a la comunidad universitaria por parte del personal administrativo, tampoco se contaban con políticas claras que motiven el desarrollar acciones en pro de contribuir y comprender las problemáticas psicosociales que aquejan al entorno social. 
Ante la presente realidad se desarrollaron herramientas, primero para que el personal administrativo intratópico pueda tener conocimiento de forma clara, las formas de organización académica, administrativa (en ese caso se plasmaron de forma gráfica el calendario académico y económico las mismas fueron socializadas a las autoridades pertinentes. Así mismo se desarrollaron nuevos canales y formas de comunicación interna, como ser tener grupos de WhatsApp, se generaron nuevos formatos de horas de ruta, se comenzó a generar actas de todas las reuniones que se desarrollaban. Se organizaron al menos 2 capacitaciones semestrales, dirigidas exclusivamente para el personal administrativo, capacitaciones que permitan mejorar el manejo de las herramientas de ofimática, como también el manejo de sus habilidades sociales, para una mejorar atención a las personas. Una capacitación fundamental que se tuvo en esta primera área de intervención, fueron las relacionadas a generar un mayor compromiso consigo mismos como también con la universidad, capacitaciones en donde se abordaron temáticas como la misión, visión institucional, comprensión de la "minoría creativa", además de mejorar las habilidades verbales y no verbales, entre otros.

Los facilitadores de los procesos de capacitación fueron identificados tanto dentro de la institución como fuera, esto con el objetivo de reforzar ciertas deficiencias o problemáticas que generaban ciertas dificultades a la hora de responder una solicitud de apoyo de los docentes o estudiantes, que buscaban desarrollar acciones en pro de la gestión de conocimientos.

También se planificaron procesos de capacitación, implicando a representantes del Ministerio de Educación, con quienes se abordaron los temas en relación con el uso de la tecnología informática y al manejo de plataformas bibliográficas como el Cauurii. Plataforma impulsada por el mismo ministerio, para poder tener fuentes de información diferenciada, con el objetivo de promover investigaciones. Esta capacitación, de forma tradicional solamente se promovía dentro de la Usfa Sede Central, en el personal docente. Saliendo se este esquema, como parte del presente trabajo investigativo, la capacitación primero se generó en el personal administrativo, con el fin de que ellos, una vez que tengan este conocimiento, puedan motivar, coadyuvar, orientar a los docentes y estudiantes su utilización.

2. Gestión con la comunidad docente, gestión que implicaron procesos de dinamización docente a partir de:

a) Reuniones de confraternización, festejo de cumpleaños, encuentros deportivos, lo que permitió generar una nueva dinámica en las relaciones interpersonales entre docentes, pudiendo identificar las fortalezas que tenía cada uno de ellos, generando verdaderos equipos de trabajo y de apoyo entre docentes, estructurando relaciones que permitió dejar de lado lo que estaba mutilado (confianza entre ellos mismos), articular lo que estada disjunto (confianza en el otro docente) pensar lo que estaba oculto (reconocer habilidades, destrezas tanto de uno mismo como del otro). Estas acciones no solamente permitieron fortalecer lazos entre el personal docente, también influenció a tener una relación más estrecha y de confianza con el personal administrativo. Convirtiendo el espacio de la universidad, como un lugar de trabajo confiable y amigable.

b) Procesos de capacitación específica a la carrera en donde se encuentran enseñando, como caso particular se tiene en carrera de psicología, donde se invitaba a personas expertas, a representantes de instituciones, para que transmitan su experiencia y estos puedan ser tomados en cuenta por los docentes en sus actividades prácticas y de interacción.

c) Procesos de capacitación en temáticas relacionadas a las características del entorno sociocultural, también 
específicas a cada una de las carreras. Capacitaciones que se desarrollaron, fruto de la organización dentro de la universidad, como también capacitaciones generadas por otras instituciones, a las cuales se les invitó a los docentes a que participen, ya sea solos o implicando a sus estudiantes

d) Finalmente se contemplaron capacitaciones específicas a la dinámica misma de la Educación Superior, en temáticas como: diseños micro curriculares, nuevos modelos de planificación de aula, estrategias didácticas, entre otros. Cada una de las capacitaciones se desarrollaban al inicio de cada gestión académica, para que el docente, en base a los conocimientos adquiridos de las capacitaciones, pueda plasmarlas en sus Planificaciones de Aula.

3. Gestión dinámica y participativa con la comunidad estudiantil, gestión que se enfocó a desarrollar nuevas formas y alternativas de comunicación con el personal administrativo, que implicó:

a) En primera instancia es menester saber que en un entorno universitario existen una diversidad de realidades socioculturales, con diversas formas de pensar y actuar, por lo que se vio por conveniente generar varios canales de comunicación, basadas desde lo tecnológico hasta un tipo de comunicación tradicional, la carta (por escrito); contemplando además el tipo de información o asesoramiento que requerían.

b) Promover, dentro del personal administrativo, una comunicación abierta, cordial, basada en lo recíproco y cordialidad, la ética y transparencia, así mismo en la puntualidad, todos estos con el fin de generar un estado de conformidad y satisfacción por parte del estudiante, en relación con su solicitud, cualquiera que fuese.

c) Dinamizar a la comunidad estudiantil mediante actividades extraacadémicas, que vayan a fortalecer tanto sus conocimientos como sus competencias; actividades planificadas y gestionadas a partir de los requerimientos de personal, identificadas en reuniones con instituciones aliadas, como también con la revisión continúa de las ofertas de trabajos en medios impresos, como es el periódico.

d) Generación de capacitaciones trimestrales, considerando temas como estilos de comunicación y formas de comunicación, enfocados a mejorar la atención al cliente, estos procesos de capacitación enfocados al personal administrativo, a favor de la comunidad estudiantil.

e) Se generaron carpetas de orientación estudiantil, para cada uno del personal administrativo intratópico, que facilite la orientación y asesoramiento al estudiante

4. Los vínculos generados con el personal de servicios también fueron importantes y aportaron a procesos de cambio en el tipo de servicio que desarrollan:

a) Se generaron reuniones semanales de evaluación, valoración y recomendaciones continuas, esto con el fin de la mejora continua y de la preservación de los ambientes universitarios.

b) Reuniones promovidas con la participación de todo el personal administrativo intratópico, para poder contemplar la valoración en cuanto a la limpieza de todos los ambientes de la universidad.

c) Generación actividades de esparcimiento con familiares del personal de servicio, además de implicarles en reuniones de confraternización, esto también coadyuvó a generar un sentimiento de pertenencia, por ende, mayor compromiso a la hora de cumplir con sus responsabilidades.

d) Todo el proceso generado con el personal de servicio se resumiría en "ser tomado en cuenta", en la dinámica interna 
administrativa como de esparcimiento dentro de la universidad.

5. Se desarrollaron actividades que permitieron tener nuevos contextos de interacción entre la comunidad docente con la comunidad estudiantil, a partir de actividades culturales, artísticas y de esparcimiento. Se tuvieron actividades de teatro, proyecciones de cine y exposición de fotografías, que permitieron un intercambio de opiniones como de criterios, fuera de lo tradicional, en espacios en donde permitió a los docentes, conocer de forma diferente al estudiante, en muchos casos estas actividades permitieron "darse cuenta" de algunas fortalezas, habilidades que tenían los estudiantes, pero que no era visible dentro del espacio tradicional de interacción áulico.

6. Se promovieron acciones que permitieron generar un vínculo entre el personal de servicio (limpieza, de seguridad, portería) con la comunidad estudiantil, esto a partir de actividades de sensibilización en cuanto al cuidado del medio ambiente, de los predios universitarios, en donde ante cualquier inconveniente de limpieza de algún predio o falla en el sistema eléctrico, fluvial, etc. la comunidad estudiantil pueda comunicar o pedir al personal de servicio el arreglo inmediato, sin necesidad de esperar y la gestión pertinente para el arreglo, obviamente todo regulado por el personal administrativo, que supervisa, de forma continua el estado de los predios de la universidad.

7. Se desarrollaron actividades que admitieron la comunicación continua, constante entre la comunidad docente y la sociedad civil en general. A partir de las gestiones pertinentes que permitió a los docentes estar de forma continua, en procesos de actualización a partir de su participación en foros, presentación de investigaciones, estudios, presentación de hojas de ruta, resultados de gestiones, participación de talleres, ferias educativas, entre otros. Esta relación entre comunidad docente y las organizaciones, entidades tanto estatales como privadas, permitió además generar en el docente, un sentimiento de pertenencia generó que éste esté agradecido con la universidad, por las diversas oportunidades que se le brinda para poder actualizarse, aprender en pro de formación.

8. Como en el caso de los docentes, de la misma manera se procedió con la comunidad estudiantil, en donde se desarrolló gestiones para promover procesos participativos continuos de los estudiantes en los diversos eventos académicos, extraacadémicos (artísticos, seminarios, talleres, etc.) en donde el estudiante al sentir que se encuentra representando a la universidad, generó en él mayor compromiso en sus estudios, en su formación. El estudiante tuvo oportunidades de involucrarse con la institución o entidad organizadora, permitiéndole además abrirse, en algunos casos, en el ámbito laboral por los tipos de aportes que desarrollaba. Así mismo permitió a los estudiantes, reaprender algunos aprendizajes, fruto del análisis de la realidad, con los conocimientos ya adquiridos a partir de su formación.

9. Él último proceso relacional que se desarrolló, fruto de la aplicación de nuevos principios basados en complejidad y transdisciplinariedad, se dio énfasis a generar reuniones, asambleas, foros, encuentros entre la sociedad civil, fundaciones, instantes estatales, con toda la comunidad universitaria, implicando desde el personal administrativo, comunidad docente, comunidad estudiantil, inclusive personal de servicio. Esto a partir de temáticas transversales que, coadyuvan la vida en armonía entre la sociedad, como es el caso de las temáticas de sensibilización a la violencia intrafamiliar, violencia sexual infantil, consumos de drogas, discapacidad. También se organizaron acciones que permitió sensibilizar sobre el buen uso del tiempo libre, a partir de la organización de proyecciones de video, teatro y exposiciones de obras de arte (fotografías, pintura, etc.). buscando siempre innovar y nuevos espacios, temas que permita la vida en armonía entre toda la comunidad universitaria, en relación con la sociedad.

\section{Conclusiones}

Después de generar todo un plan de intervención basado en la dinamización del contexto/realidad académica con el contexto/realidad 
sociocultural a través del proceso de gestión del conocimiento, permitió en la universidad contar con un equipo cohesionado, con mayor sentimiento de pertenencia en su persona, permitió una mayor amplitud en la toma de decisiones a nivel intratópico, permitió convertir a la universidad, como espacio de referencia en el municipio alteño, entro otros cambios.

Dentro de las certezas de la presente investigación, se identifica que la importante de considerar el contexto/realidad académica y el contexto/realidad sociocultural, mediados por el proceso de gestión del conocimiento, como importantes en la formación profesional, basada en innovación, creatividad, promoción de procesos participativos e involucramiento de toda la comunidad universitaria con las entidades sociales, municipales, locales, etc.

Entre las inquietudes se destacan: creciente interdependencia e interacción entre personas, instituciones, corporaciones, tanto privadas como estatales en el ámbito de la educación superior, ha traído cambios significativos en las percepciones del mundo y con ellas importantes desafíos al llamado proceso de globalización ¿Cómo responde la educación Superior a los continuos/coyunturales desafíos locales y globales? (¿estaríamos considerando de un proceso global? ¿Cómo fortalecer el desarrollo de habilidades y actitudes de cooperación, pensamiento crítico, responsabilidad compartida, comunicación, participación efectiva?

Para un proceso de gestión del conocimiento, es importante poder considerar diversos canales y formas de comunicación, que permitan la viabilidad de la información que se quiera transmitir entre los integrantes de la Universidad, cada uno de los canales y formas de comunicación deben ser de conocimiento de todos los miembros de la comunidad universitaria, que abarcan desde una simple conversación entre los interesados hasta un proceso formativo formal.

Así mismo es importante generar nuevos y constantes vínculos con entidades sociales, privadas, estatales (a nivel local, municipal, departamental y central) que, a partir de reuniones, actividades conjuntas, dar apertura a la puesta en práctica de los conocimientos adquiridos por parte del estudiante, como también que permita, al docente, extraer, fruto de la experiencia previa, nuevos como actualizados conocimientos. Ahora bien, estos nuevos conocimientos, deben ser religados con experiencias para su respectiva valoración, proceso que se da de forma continua y circular.

A partir de la gestión del conocimiento, aplicado en el ámbito de la Educación Superior, específicamente en la Universidad Privada San Francisco de Asís, permitió, entre los implicados, generar y reforzar un sentimiento de pertenencia, que a la vez influenció para poder generar un continuo proceso de gestión del conocimiento, a partir de la participación activa de actividades con y desde la comunidad.

El proceso de gestión del conocimiento debe ser analizado, se hace necesario encaminar, reencaminar para poder ser promovida de forma continua por las autoridades universitarias, considerando que, al tener una posición estratégica, como lo sostiene el investigador, facilita una gestión continua, participativa y activa; ya que logra implicar a todos los subsistemas que conforma la universidad, teniendo como resultado, además, el del mejoramiento de los vínculos y tipos de comunicación entre los sujetos que están implicados con la universidad.

\section{Referencias}

Alavi M. \& Leidner, D. (1999) Sistemas de Gestión del conocimiento: cuestiones, retos y beneficios. España.

Andreu. R. (1999). La gestión integral del conocimiento y del aprendizaje. en Economía Industrial, núm. 326, pp. 63-72

Arroyave, D. (2013) La educación planteada desde una estrategia de pensamiento complejo: una apuesta pedagógica para re-pensar la Universidad XXI. Observaciones de Reformas Universitarias, Francia

Arroyave, D. (2013), Seminario Educación Compleja. Documento del Doctorado en Educación con enfoque en la complejidad y la Investigación Transdisciplinar. Escuela Militar de Ingeniería.

Barney, J. B. (1991). Firm resources and sustained competitive advantage. Journal of Management. (vol 27)

Devlin, K. (1999). Infosense: Turnin Information in Knowledge. NY W.H. Freeman

González, J. (2013). Aula Mente Social Pensamiento Transcomplejo. Universidad Simón Bolívar.

González, J. (2013). Currículo Transcomplejo. Universidad Simón Bolívar.

Kogut, B. \& Zander, U. (1992). Knowledge of the firm: combinative capabilities, and the replication of technology. Organization Science, 3(3). 
López, D. (2006). El conocimiento y la comunicación: dos pilares fundamentales de la organización de la sociedad de la información. 9(2),91-109. ISSN: 0122-8285. Recuperado de: https://www.redalyc.org/articulo.oa?id=649/64900209

Machado, A. (1998). Caminante, no hay camino. Planeta.

Morín, E. (2000). La menta bien ordenada. Ediciones Seix Barral.

Morin, E. (2003). El método V. La humanidad de la Humanidad. La identidad humana. Cátedra.

Morin, E. (2005). Epistemología de la complejidad. Recuperado de: www.pensamientocomplejo.com.

Morin, E. (1984). Ciencia con conciencia, Anthropos.

Nonaka, I. \& Takeuchi, H. (1995). The Knowledge-Creating Company, Oxford University Press, ISBN 0-19- 509-269-4.

Panza, N. (2020). Docente universitario, alfarero de vidas...Revista Tecnológica-Educativa Docentes 2.0, 8(1), 60-65, https://ojs.docentes20.com/index.php/revista docentes20/article/view/106

Rodríguez, G., Gil, J.\& García, E. (1996). Metodología de la investigación cualitativa. Ediciones Aljibe.

Rozo, T (2003) La construcción del conocimiento desde el hombre, la sociedad, la vida y el mundo. Universidad de Sonora.

Sotolongo, P. (2006). La revolución contemporánea del saber y la complejidad social: hacia unas ciencias sociales de nuevo tipo Buenos Aires. Consejo Latinoamericano de Ciencias Sociales.

Tobon, S. (2005). Secuelas didácticas en el contexto de la gestión educativa universitaria, Editorial Pearson. 